\title{
Impact of Viral Etiologies on the Development of Novel Immunotherapy for Hepatocellular Carcinoma
}

\author{
Chun Jye Lim, BSc ${ }^{1}$ Valerie Chew, $\mathrm{PhD}^{1}$ \\ ${ }^{1}$ Translational Immunology Institute (TII), SingHealth-Duke-NUS \\ Academic Medical Centre, Singapore, Singapore
}

Semin Liver Dis 2020;40:131-142.

\begin{abstract}
Address for correspondence Valerie Chew, PhD, 20 College Road, The Academia, Level 8 Discovery Tower, Singapore 169856, Singapore (e-mail: valerie.chew.s.p@singhealth.com.sg).
\end{abstract}

\begin{abstract}
Keywords

- hepatitis virus infection

- hepatocellular carcinoma

- immunotherapy

- tumor microenvironment

- viral-related HCC

Hepatocellular carcinoma (HCC), is the most common type of liver cancer which is derived mostly from the background of chronic inflammation. Chronic hepatitis viral infection remains one of the most common etiologies implicated in chronic liver inflammation, cirrhosis, and HCC. With such background inflammation, immunotherapy-particularly the checkpoint inhibitors-have been tested in HCC patients with unprecedented success. However, despite the initial enthusiasm, the response rate to immunotherapy remains modest in most clinical trials (approximately 20\%), with mixed reports on response rates in hepatitis viral-related HCC as compared with nonviral HCC. Given such complexity in response to immunotherapy, it is increasingly appreciated that deeper understanding of the tumor molecular features and tumor microenvironment of hepatitis viral-related HCC is crucial for the design of more effective immunotherapeutics. We discuss herein the current knowledge in tumor genomic mutational and immune landscapes as well as the ongoing immunotherapy trials in HCC with the unique focus on their viral etiologies. Based on this understanding, we also outline perspectives and rationale on the design of potential immunotherapeutic strategies in HCC patients according to their viral etiologies.
\end{abstract}

Hepatocellular carcinoma (HCC) remains the fifth most commonly diagnosed cancer and second leading cause of cancer deaths for males worldwide. ${ }^{1}$ Viral hepatitis remains one of the major etiologies for HCC, particularly hepatitis B virus (HBV) chronic infection, which has a high prevalence in Asia and Africa coinciding with the high incidence of HCC in these areas. ${ }^{2}$ Currently, chronic infection with hepatitis C virus (HCV) is one of the main cause of HCC in the Western world. ${ }^{1}$ Besides that, hepatitis D virus (HDV) co-infection with chronic HBV has also received increased awareness and has been shown to significantly increase the risk of HCC. $^{3}$ Development of HCC in the background of chronic inflammation, particularly that driven by chronic hepatitis virus infection, has been reported to be a result from an immunosuppressive and exhausted tumor microenvironment (TME). This makes viral-related HCC a potential candidate for immunotherapy, which aims to reactivate the exhausted local antitumor immunity. However, many challenges remain for this strategy. Recent reports on clinical outcome from the immunotherapy trials, mostly involving checkpoint inhibitors (immune checkpoint blockades), have been modest.

Given the complexity and dynamic interaction between the tumor cells and immune microenvironment, this review aims to explore the current understanding on viral-related HCCs, based on its unique tumor mutational landscape versus immune microenvironment and its impact on the development of novel immunotherapeutic strategies.

\section{Understanding HCC Based on Viral Etiologies}

As immunotherapy is based upon the basic principle of the immune system recognizing and targeting the tumor cells as foreign, understanding both the tumor antigenicity and the immune microenvironment is essential for a successful immune-based therapy. Are the tumor antigens immunogenic in HCC? Are they being presented and recognized? Is 


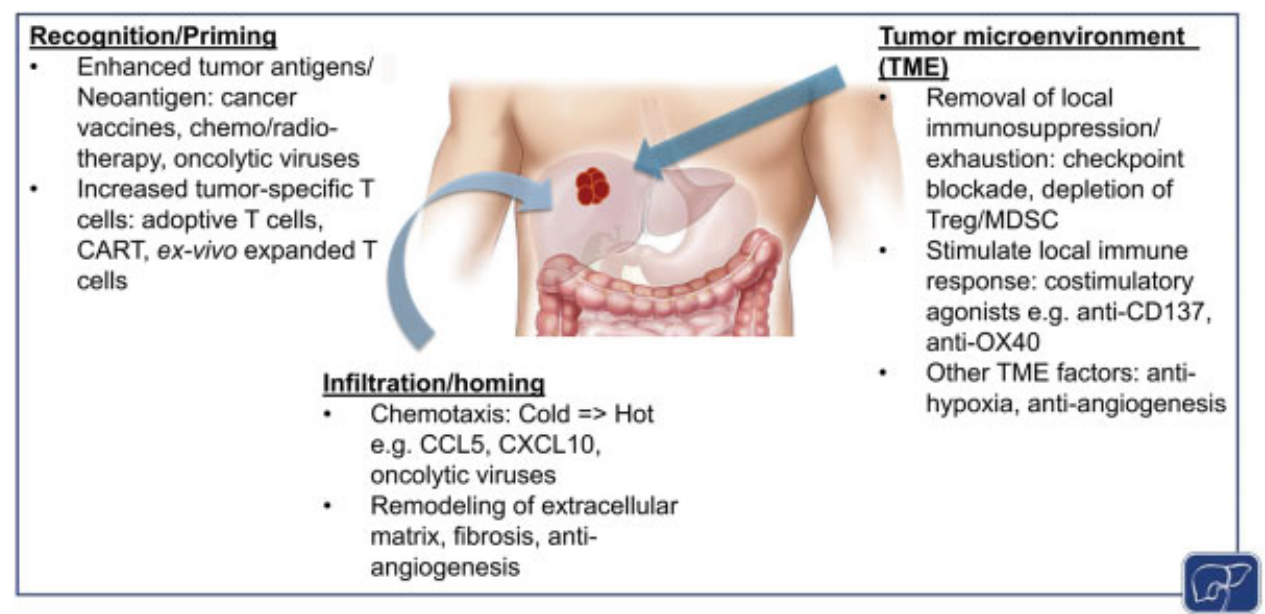

Fig. 1 Elements of a successful immunotherapeutic strategies. Three key elements of successful immunotherapeutic strategies with enhanced antitumor immunity include: (1) to enhance recognition of tumor antigens or the priming of immune system, i.e., using T-cell therapy; (2) to stimulate cytotoxic immune cells infiltration or homing to the tumor mass such as using therapies enhancing T-cell chemoattractants; and ( 3 ) to modulate the tumor microenvironment such as using check-point inhibitors to reverse the immune exhaustion in the tumor.

there sufficient tumor infiltration of cytotoxic immune cells and are they able to target and kill the tumor cells? Is the immune microenvironment conducive for immunological cytotoxicity against the tumor cells? Many of these areas are essential and can be modulated to ensure a successful immunotherapeutic strategy (-Fig. 1). As it is a complex ecosystem, tailoring a successful immunotherapy is more challenging than one would have thought. We would herein discuss these aspects of immunotherapy, starting from tumor mutational landscapes to immune microenvironment, in the highly heterogeneous HCC particularly focusing on viral etiologies.

\section{Mutational Landscapes in Viral-Related HCCs}

As mentioned, one of the key principles of immunotherapy is the recognition of tumor antigens by the immune system as foreign. ${ }^{4}$ In the case of viral-driven HCC, the recognition of viral-related antigens or the antigens derived from the viral-induced mutations could then serve as a basis for immunotherapy.

Hepatitis B virus is a double-stranded DNA virus which integrates with the host human genome while HCV is an enveloped single-stranded RNA virus which does not show DNA integration. ${ }^{5}$ HBV viral proteins that could be expressed during its replication cycle include hepatitis B surface antigen (HBsAg), hepatitis B core antigen ( $\mathrm{HBcAg})$, and hepatitis $\mathrm{B} X$ antigen (HBxAg). ${ }^{6}$ For instance, it was previously proposed that HBV-encoded HBx protein plays multiple oncogenic role associated to hepatocarcinogenesis. ${ }^{7}$ It was however, long speculated that HBV-related proteins may not be a good tumor antigen target due to the clearance of hepatitis-related proteins observed in HBV-related HCC. ${ }^{8,9}$ However, recent studies have demonstrated that it is possible to target $\mathrm{HBV}$-human chimeric proteins produced as a result from HBV-human genome integration. ${ }^{10,11}$ In fact, recent attempts to treat $\mathrm{HBV}$-related $\mathrm{HCC}$ by targeting HBV antigen resulted in promising benefit. ${ }^{12,13}$
On the other hand, HCV viral genomic RNA encodes for a polyprotein, which is processed by host and viral proteases into at least 10 different proteins. ${ }^{14}$ There is currently no $\mathrm{HCV}$ vaccine available due to many challenges in vaccine development including high variability of HCV virus and the lack of suitable in vitro and in vivo models. ${ }^{15}$ Henceforth, an HCV antigen targeting immunotherapy for HCV-related HCC would most likely face similar challenges. Instead, HCV infection was traditionally treated with interferon-based therapy, ${ }^{16}$ until recent development of direct-acting antiviral (DAA) agents with high efficacy and safety profile. ${ }^{17,18}$ However, HCV eradication was controversial, ${ }^{19}$ following a series of reports on unexpected higher incidence or recurrence of HCC in DAA-treated HCV patients. ${ }^{20-22}$ Although it was later concluded that patient selection may play a critical role. ${ }^{23}$ There is also currently limited HDV-targeted therapy available apart from general antiviral strategy. Therefore, the most promising strategy of targeting viral-specific antigens for the treatment of HCC will be on HBV-specific antigens.

It is also not known if any of the viral-driven mutations lead to expression of immunogenic antigens which could be targeted by immunotherapy. For instance, several genes were reported to be altered due to HBV-DNA integration events such as putative cancer-related TERT, MLL4, and CCNE1 genes. ${ }^{24} \mathrm{~A}$ more recent comprehensive genomic atlas of HCC reported on two major HBV integration sites with recurrent mutations: MLL4 and TERT; while HCV-related HCC displayed higher frequency of CDKN2A promoter silencing and TERT promoter mutation. ${ }^{25}$ In addition, EGF was also implicated in HCV cirrhosis and $\mathrm{HCV}$-related $\mathrm{HCC}^{26,27}$ while immune-related gene Interleukin-1 $\beta$ (IL-1 $\beta$ ) was reported to be associated with HCC in Japanese patients with chronic HCV infection. ${ }^{28}$ These studies delineate genetic alterations in hepatitis viralrelated HCC; however, there were no specific studies to determine how antigenic these hepatitis viral-related mutations are.

Furthermore, it was reported and further validated that HBV-induced tumorigenesis is in fact a result from the immune-mediated liver damage $\mathrm{e}^{29-31}$ or dysfunction of 
cytotoxic immune cells, rather than as a result of these viralrelated oncogenes. ${ }^{32}$ Several genome-wide association studies have also linked multiple HBV-induced genes to immune functionality such as HLA variants, MICA, CTLA4, and IL-6 in the predisposition and prognosis of patients with HBVassociated HCC. ${ }^{33-36}$ Likewise, HCV-induced inflammation and eventual immune dysfunction both play a major role in carcinogenesis of HCV-related HCC. ${ }^{37}$ A genome-wide association study in HCV-induced HCC identified a susceptibility locus in the $5^{\prime}$ flanking region of MICA that was associated with lower soluble MICA proteins levels and development of HCC from chronic HCV infection. ${ }^{38}$ MICA $^{38}$ binds to NKG2D and results in the activation of NK cells and T cells. ${ }^{39}$ Its downregulation in both HBV and HCV-related HCC would indicate a mechanism of hepatitis viral-induced immunosuppression. It is therefore likely that the immunosuppressive microenvironment of viral-related HCC would make a better therapeutic target as opposed to the viral-specific mutations or antigens.

Besides specific mutations, it was previously reported that total mutational burden could be an indication of response to the checkpoint blockade in multiple cancer types. ${ }^{40,41}$ The total mutational burden of HCC is moderate, considerably higher than pancreatic or prostate cancer but lower than major cancers like lung cancer and melanoma, both of which respond better to the checkpoint inhibitor than HCC. ${ }^{42}$ In fact it was reported that HBV-related HCC harbors lower total mean mutation rate than that of non-HBV-related HCC whereas there was no significant difference in the pattern of somatic mutations in HBV-, HCV-, and non-HBV/non-HCV-related HCC. $^{43}$ This may indicate a lower response rate in HBV-related HCC, which in fact was reported to be the case in CheckMate040 clinical trial using anti-PD-1 antibody nivolumab in advanced HCC ( - Table 1). ${ }^{44}$ However, given the heterogenous nature of HCC with multiple different molecular classifications $^{45}$ as well as intratumoral heterogeneity, ${ }^{46}$ it remains challenging to generalize on the response to immunotherapy based on the current understanding of genomic landscape of viral-related HCC. Indeed, deeper understanding in the tumor immune microenvironment would be essential to gain better insights in this complex mechanism.

\section{Tumor Immune Microenvironment}

The critical role of immune microenvironment in HCC progression or disease prognosis was previously discussed. ${ }^{42,47-49}$ Most of the immunoprofiling of TME in viral-related HCC was performed compared to healthy livers or the non-HCC chronic viral-infected livers. ${ }^{50}$ For instance, Treg accumulated in TME of HBV-related HCC, as compared with nontumor and healthy liver controls, has been shown to associate with impaired CD8+ T cells, disease progression, and poor survival in HCC patients. ${ }^{51}$ In fact, the role of Treg in maintaining immune tolerance during chronic HBV infection has been previously shown $^{52}$ and its accumulation was linked to HBV-induced TGF- $\beta$-miR-34a-CCL22 signaling pathway. ${ }^{53}$ Dysfunctional $\mathrm{CD} 8+\mathrm{T}$ cells with upregulation of multiple exhaustion markers such as PD-1, Tim-3 or Lag-3 were also previously described in chronic HBV infection ${ }^{54}$ or HBV-related HCC. ${ }^{5,56}$ It was reported that HCV-specific CD8+ T cells express various inhibitory receptors, including CTLA-4 and PD-1 with enhanced exhaustion phenotype that could be reactivated by $\mathrm{PD}-1 / \mathrm{PD}-\mathrm{L} 1$ blockade. ${ }^{5,58}$ Whereas, the immunosuppressive function of Treg could be fine-tuned by OX40 in HCV-infected liver tissues. ${ }^{59}$

Dysfunction of other lymphocytes such as the reduced cytotoxicity of NK cells was also reported during chronic hepatitis viral infection. ${ }^{60}$ It was previously speculated that NK is more dysfunctional in chronic HBV as compared with chronic HCV infection. ${ }^{61}$ However, MICA mutation, which is important for $\mathrm{T}$ and $\mathrm{NK}$ cells activation, ${ }^{34,38}$ was detected in both HBV and HCV-related HCC. In fact, NK cells impairment was previously shown in both $\mathrm{HBV} / \mathrm{HCV}$-related $\mathrm{HCC}^{62}$ rendering NK cell therapy an attractive option for viral-related HCCs (-Fig. 2). As for myeloid subsets, the resident macrophages in liver, the kupffer cells, were known as one of the important immune cells involved in multistep antiviral immunity and disease pathogenesis during HBV and HCV infection. ${ }^{63}$ It involves a cascade of events with IL-6 and TNFa-driven chronic inflammation and compensatory proliferation as well as oxidative stress which eventually drives the process of carcinogenesis. ${ }^{64,65}$ Such viral-driven, chronic, and unresolved inflammation is in fact a well-recognized mechanism in HCC development and progression. ${ }^{66}$ In fact not only the inflammatory functions of these macrophages, the tolerogenic and suppressive M2 macrophages were also shown to be important in tumor progression and invasiveness via CCL22-mediated epithelial-mesenchymal transition. ${ }^{67}$ More importantly, It was also shown that macrophages could impair T-cells immunity via enhanced PD-L1 expression in HCC tumors. $^{68}$

A recent high dimensional immunoprofiling of TME with cytometry by time-of-flight comparing HBV-related versus nonviral hepatitis-related HCC demonstrated higher density of intratumoral immunosuppressive Treg and $\mathrm{T}_{\mathrm{RM}}$ in HBVrelated HCC. ${ }^{69}$ This is consistent with previous studies that have identified higher Treg and $\mathrm{T}_{\mathrm{RM}}$ numbers in chronic $\mathrm{HBV}$ infection. ${ }^{70,71}$ The same study also reported relatively lower proportions of CD244 expressing cytotoxic NK cells in HBVrelated HCC compared with nonviral-related HCC. ${ }^{69}$ Indeed, reduced CD244 has been previously reported to associate with NK cells dysfunction and immune tolerant in chronic HBV infection. ${ }^{72}$ Most importantly, PD-1 expression was enhanced in both Treg and $\mathrm{T}_{\mathrm{RM}}$ in HBV-related HCC, consistent with a virus-induced immunosuppressive or exhausted TME. $^{69}$ In contrast, others however, described no significant difference in PD-L1 and LAG-3 expression comparing uninfected (14/29 patients) versus virus-infected (HBV: 1/29 and HCV: $14 / 29$ patients, respectively) HCC. ${ }^{40}$ Given the low number of HBV-HCC case in this study, it is hard to conclude if PD-1/PD-L1 pathways were indeed affected. A bigger cohort study will be needed to distil the differences in immune landscapes of viral versus nonviral HCC.

Overall, chronic viral hepatitis induced a chronic but yet dysfunctional, liver inflammation which eventually led to the 


\begin{tabular}{|c|c|c|c|c|c|c|c|c|c|c|c|c|c|c|}
\hline $\mid \begin{array}{l}\overline{0} \\
\frac{1}{2} \\
0 \\
0\end{array}$ & 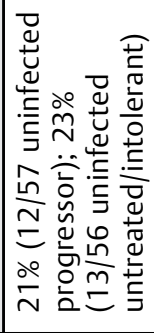 & 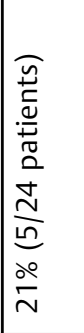 & 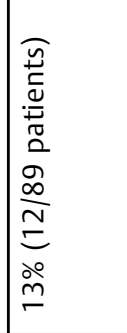 & 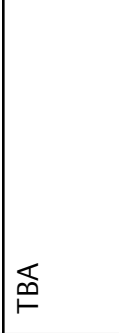 & 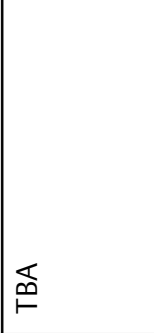 & 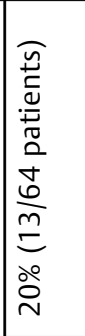 & 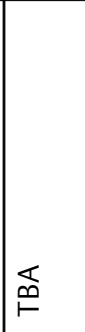 & 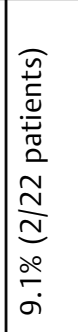 & $\Sigma$ & 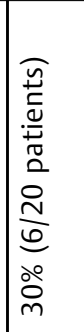 & 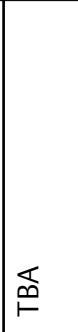 & 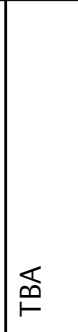 & 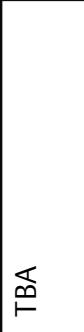 & 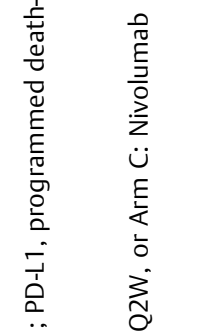 \\
\hline 壱 & 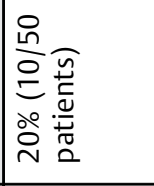 & 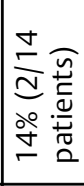 & 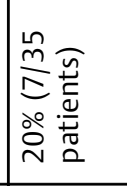 & 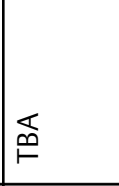 & 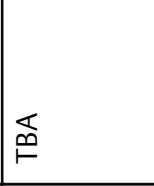 & & 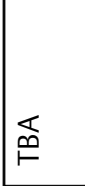 & 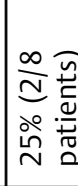 & 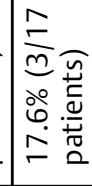 & 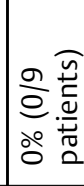 & 店 & 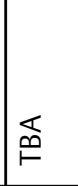 & $\underset{\wp}{\vdash}$ & 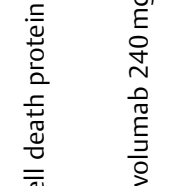 \\
\hline 帝 & 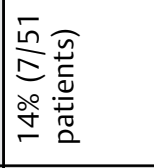 & 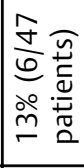 & 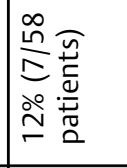 & 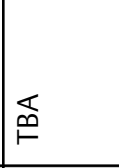 & 市 & 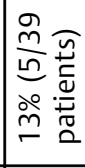 & 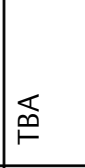 & 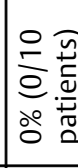 & z & 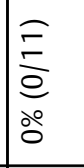 & 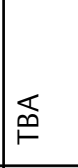 & 吕 & $\underset{\wp}{\vdash}$ & 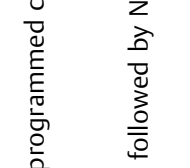 \\
\hline 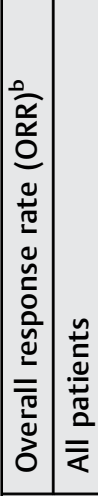 & 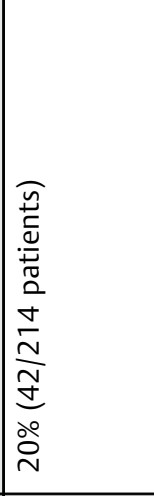 & 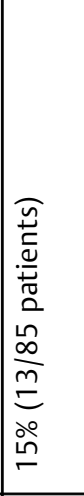 & 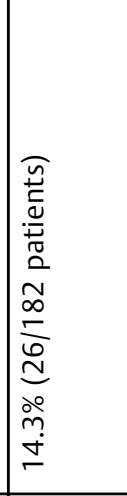 & 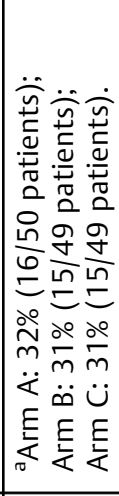 & 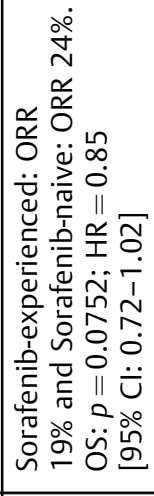 & 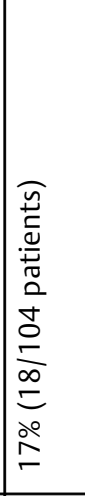 & 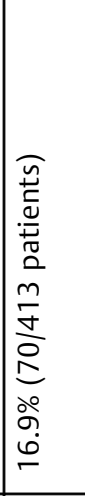 & 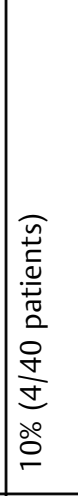 & 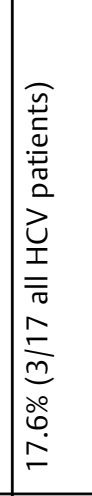 & 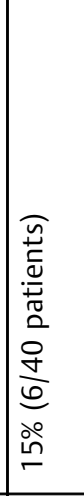 & $§$ & 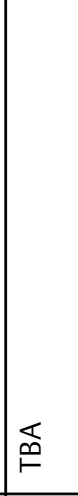 & 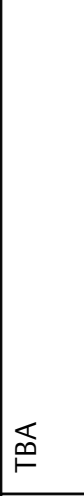 & 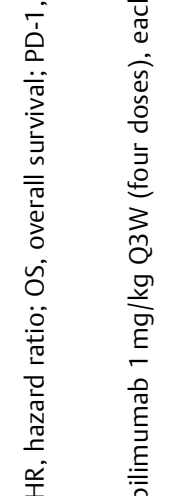 \\
\hline 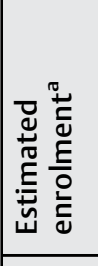 & $\stackrel{\nabla}{\sim}$ & 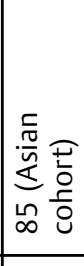 & 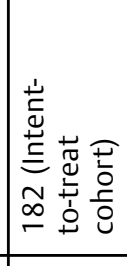 & $\stackrel{\infty}{\mp}$ & 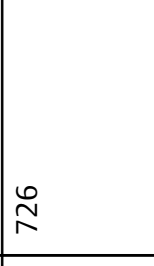 & $\underset{0}{0}$ & $\frac{m}{\sigma}$ & 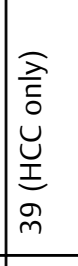 & $\stackrel{\curvearrowright}{\sim}$ & 牯 & $\begin{array}{l}\circ \\
\stackrel{\circ}{m} \\
\check{-}\end{array}$ & 吕 & 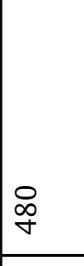 & 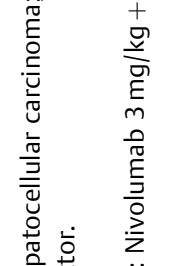 \\
\hline 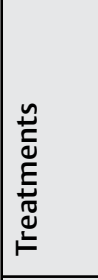 & 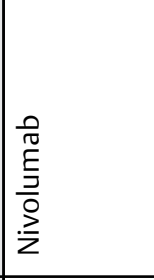 & 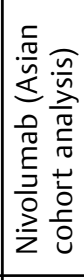 & 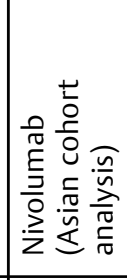 & 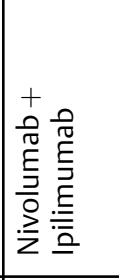 & 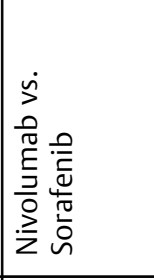 & 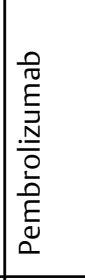 & 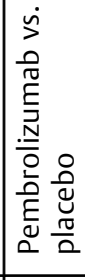 & 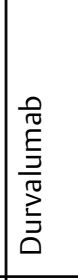 & 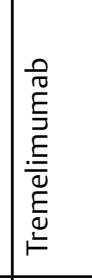 & 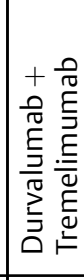 & 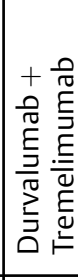 & 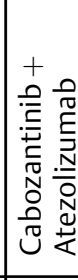 & 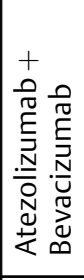 & 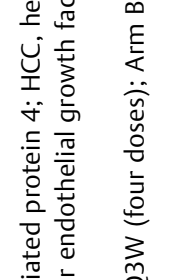 \\
\hline 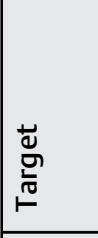 & $\bar{a}$ & 家 & $\bar{a}$ & 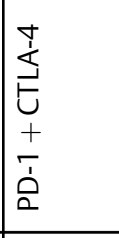 & $\bar{a}$ & ì & $\bar{a}$ & $\begin{array}{l}\overline{1} \\
\text { Q̀ }\end{array}$ & $\stackrel{+}{\stackrel{J}{\Xi}}$ & 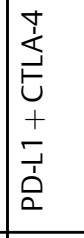 & 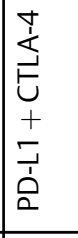 & 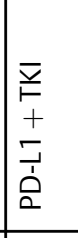 & 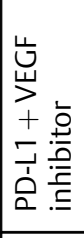 & 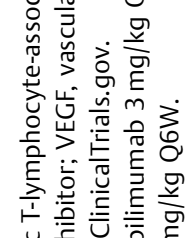 \\
\hline $\begin{array}{l}\stackrel{\Xi}{\tilde{~}} \\
\frac{c}{\alpha}\end{array}$ & $\equiv$ & $\equiv$ & $\equiv$ & $\equiv$ & $\equiv$ & $=$ & $\equiv$ & $\equiv$ & $=$ & $\equiv$ & $\equiv$ & $\equiv$ & $\equiv$ & 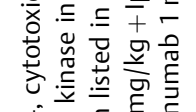 \\
\hline 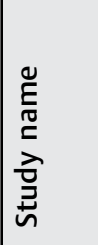 & 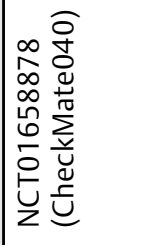 & 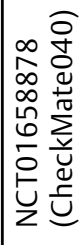 & 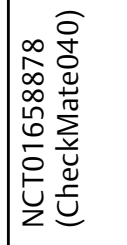 & 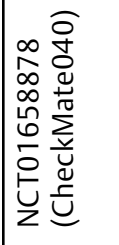 & 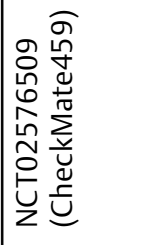 & 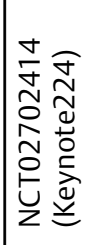 & 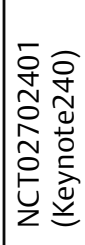 & $\mid \begin{array}{l}0 \\
0 \\
10 \\
\tilde{o} \\
6 \\
0 \\
. \\
z\end{array}$ & $\begin{array}{l}\infty \\
\stackrel{\infty}{n} \\
\tilde{\infty} \\
o \\
0 \\
0 \\
0 \\
\varrho \\
z\end{array}$ & 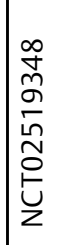 & 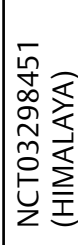 & 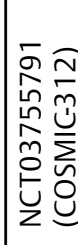 & 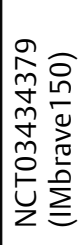 & 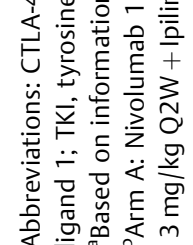 \\
\hline
\end{tabular}




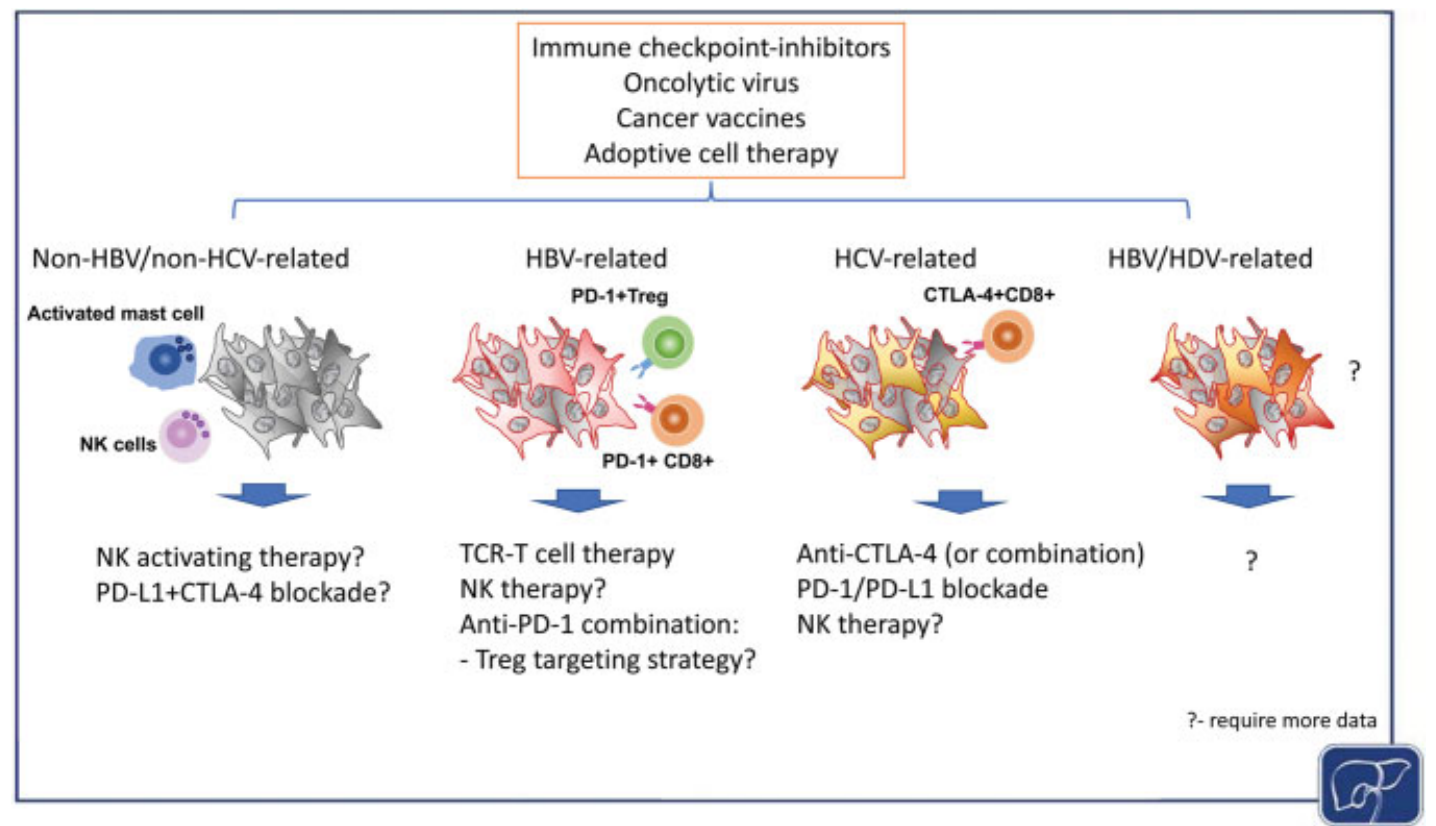

Fig. 2 Immunotherapy in hepatocellular carcinoma (HCC) with perspectives on viral hepatitis-related HCC. General immunotherapies tested in viral- and nonviral-related HCCs include immune checkpoint-inhibitors, oncolytic virus, cancer vaccines, and adoptive cell therapy with various success. The previously reported microenvironments of viral-related and nonviral-related HCC were summarized based on enriched immunesubsets. Suggested immunotherapies were listed below based on the current knowledge on their respective microenvironments even though more data are required in some of these areas (marked with a question mark).

development of HCC. The resulting immune exhaustion and multiple layers of immune dysregulation provide unique opportunity for immunotherapeutic intervention. Despite this, the complex immune landscapes and interaction between different immune subsets hindered the success of one single immunotherapeutic agent. This is indeed reflected in the seemingly lower response rate of HBV-related HCC to checkpoint blockade (-Table 1). Furthermore, given the complex etiopathologies of HCC including alcohol-induced and nonalcoholic fatty liver disease (NAFLD), most of which also induce a chronic inflammation in the liver, it will hence remain challenging to clearly define the immune landscapes of HCC according to its etiologies.

\section{Potential Novel Immunotherapies Based on Viral Etiologies in HCC}

With the success of phase II clinical trials using checkpoint inhibitor anti-PD-1 in HCC (CheckMate040 and Keynote224), there has been increasing interest and use of immunotherapy in HCC. ${ }^{44,73}$ Despite the fact that phase III Checkmate459 and Keynote240 did not reach predetermined endpoints, the clinical benefits of immunotherapy in HCC were well accepted in the field. Several immunotherapeutic strategies for HCC treatment, in particular the combination checkpoint-inhibitor immunotherapy, are currently under various phases of ongoing clinical trials. Besides that, several other immunotherapies such as adoptive cell therapy and cancer vaccine are also in different phases of development. We will discuss these immunotherapies in the light of hepatitis-related HCC, based on the limited data available.

\section{Engineered T-Cell Therapy}

$\mathrm{T}$ cell engineered to express chimeric antigen receptors (CARs) or autologous $\mathrm{T}$ cells expanded and engineered ex vivo with specific targeted tumor antigen(s) have been used for the treatment of HCC. For instance, there are several ongoing phase I or phase I/II trials using CAR-T cells directed against GPC-3, CEA, or Mucin 1 in various solid tumors including $\mathrm{HCC}^{74}$ It was also shown that T cell could respond to HLA-A2-restricted tumor antigens such as NY-ESO-1 and SSX-2. ${ }^{75}$ T-cell therapy targeting HCC-specific antigen such as $\alpha$-fetoprotein (AFP) was explored previously without much success (NCT03349255). Indeed, most of the tumorspecific $T$ cells had low affinities and expressed higher PD-1 T-cell exhaustion marker. ${ }^{75}$ The highly heterogeneous nature of HCC cells also suggests that immunotherapy targeting a single tumor antigen would likely not be successful.

On the other hand, targeting HBV-related antigen in HBVrelated HCC is challenging as chronic HBV infection often results in HBV proteins clearance ${ }^{8,9}$ and HBV-DNA integration or insertion into host genome leads to defective or small fragments of HBV antigens expression. ${ }^{76,77}$ HBV-specific T-cell responses were rarely detected in HCC patients ${ }^{75}$ and HBV therapeutic vaccine composed of HBsAg with a proprietary adjuvant showed little success in clearing chronic HBV infection. ${ }^{78}$ This, however, could be controversial and it was demonstrated that HBV can actually be treated with ex vivo expanded autologous $T$ cells genetically modified to express HBsAg-specific T-cell receptor. ${ }^{12}$ Even though it was demonstrated in just one case with end-stage metastatic HCC that expressed HBsAg who also received transplanted nonHBV liver, the team has confirmed its feasibility, safety as 
well as significant reduction in HBsAg levels. ${ }^{12}$ In fact, most of the HBV-DNA integration is incomplete and the insertion of viral DNA into the human genome results in the expression of HBV-human chimeric proteins. ${ }^{10,11}$ A recent study by Tan et al demonstrated a proof of principle that T-cell therapy could be tailored to target antigens from integrated HBV as a personalized TCR T-cell immunotherapy for HBV-related HCC. $^{13}$ In this study, it was shown that HBV-related HCC tumor cells expressed antigenic epitopes translated from the short integrated HBV mRNAs, that could be detected by and resulting in T-cell activation. ${ }^{13}$ Autologous $\mathrm{T}$ cells were engineered to express the selected TCRs specific for epitopes of HBV-DNA from metastases and were adoptively transferred to two HCC patients with recurrence after liver transplantation. This personalized T-cell therapy is proven to be safe in both patients with one patient showing reduction in volume of metastases during the treatment. ${ }^{13}$

For chronic HCV infection, a CAR-T against HCV targeting the HCV/E2 glycoprotein was previously constructed to control HCV infection and it was capable of secreting antiviral and pro-inflammatory cytokines and lysing HCV-infected hepatocytes in vitro. ${ }^{79}$ However, there is currently no clinical trial with such HCV-targeting CAR-T therapy and the efficacy in a clinical setting for HCV-related HCC remains to be tested. Therefore, there is a stronger current evidence support T-cell therapy in HBV-related HCC but not in HCV-related or nonviral-related HCC (-Fig. 2).

\section{Other Adoptive Cell Therapies}

Other forms of adoptive cell therapies (ACT) have been explored as immunotherapies for HCC and most of these therapies are not targeted or specific to any particular tumor antigens in HCC. For instance, treatment in $150 \mathrm{HCC}$ patients with adoptive immunotherapy using autologous TILs, expanded and activated with IL-2 in vitro, demonstrated improved recurrence-free survival (RFS) after resection. ${ }^{80}$ However, the technical challenge of isolating TILs as well as lack of specificity of the whole population of TILs without selection may limit the success of such approach.

Alternatively, cytokine-induced killer (CIK) cells, a heterogeneous cytotoxic immune population including CD8+ T cells, NK cells, and CD3 + CD56 + NKT cells, were also tested on HCC patients. CIK cells are expanded from autologous peripheral blood mononuclear cells stimulated with a cocktail of cytokines inclusive of IL-1, IL-2, IFNY, and anti-CD3 antibody. It was first demonstrated to be safe with lower recurrence rate and improved RFS in 76 treated versus 74 control HCC patients. ${ }^{80}$ Subsequently two other randomized phase III trials using CIK therapy have been shown to provide longer median time to recurrence ${ }^{81}$ or improved RFS and overall survival (OS). ${ }^{82}$

Another ACT using NK cells has also been explored as HCC immunotherapy, based on the rationale that NK cells are dysfunctional in HCC and tumor-infiltration with activated NK cells is associated with superior HCC patient survival. $^{47,60,83}$ There is only one complete feasibility and safety study using adoptive NK cells extracted from cadaveric donor liver perfusate for liver transplant recipients with HCC, which demonstrated reduced recurrence rate. ${ }^{84}$ Several other NK cell therapy trials in HCC are currently ongoing (clinicaltrials.gov). The latest development of engineered NK cells or CAR-NK cells providing tumor specificity has promising future for NK cell therapy for cancers including HCC. ${ }^{85}$ Given the lower density of NK cells in HBV-related HCC compared with NBNC-HCC ${ }^{69}$ and the impairment of NK cells in viral-related $\mathrm{HCC}^{62}$ it remains to be determined if adoptive NK therapy could actually enhance the clinical outcome in both HBV- and HCV-related HCCs (- Fig. 2). Last but not least, NK cell activating strategies such as using IL-12, IL-15, or type I interferons, would be worth exploring in nonviralrelated $\mathrm{HCC}$, given they were reported to be relatively more functional and abundant compared with the viral-HCC ${ }^{62,69}$ (-Fig. 2).

\section{Oncolytic Virus Therapy}

As an important organ receiving blood and nutrients from the guts and the center of detoxification, liver is known as an immune-tolerogenic organ. ${ }^{86}$ It is highly tolerogenic toward gut-derived bacterial metabolites entering the liver via the portal vein ${ }^{87}$ but was previously shown to illicit a robust antiviral immune response. ${ }^{88}$ For instance, it was shown that HCC progression could be controlled with the TLR3 activation, a viral-related innate immune response. ${ }^{83}$ Oncolytic virus therapy which show selective infection and killing of tumors cells has also been explored. ${ }^{89}$ These viruses can also be engineered to express genes like GM-CSF, an immunestimulatory cytokine which could enhance antitumor immunity by stimulating antigen-presenting cells such as dendritic cells and promoting infiltration and maturation of immune cells like NK cells and T cells. ${ }^{90}$ Oncolytic virus therapies were tested in preclinical trials and a few of them were tested on phase I or II clinical trials for HCC. ${ }^{91}$ One of them showed that the use of JX-594, an engineered Vaccinia virus with thymidine kinase-deactivated, was well tolerated ${ }^{92}$ and demonstrated promising outcome associated with high viral dose in phase II clinical trial on HCC patients. ${ }^{93}$ A randomized phase III trial comparing JX-594 followed by sorafenib versus sorafenib in patients with advanced HCC (PHOCUS) (NCT02562755) started in late 2015 but was recently recommended to stop after the interim futility analysis, which predicted that the study is unlikely to meet its primary objective of overall survival by the time of the final analysis. This decision was not related to the safety of the investigational product as the therapy was generally well tolerated by the patients. Despite that, other than direct killing and immunomodulating properties, oncolytic virus could also induce tumor cell death ("immunogenic cell death") and the release of tumor antigen which further enhances the antitumor immunity. However, given the immunosuppressive microenvironment of HCC, it is most likely that the success of oncolytic virus could be enhanced in combination with immune checkpoint blockades. Indeed, several clinical trials using the combination of oncolytic virus and immune checkpoint inhibitors are ongoing, including in advanced HCC. ${ }^{94}$ 
There is currently no information on whether oncolytic virus would work better or worse in viral-related HCC. However, oncolytic virus JX-594 has been shown to have an effect on suppression of hepatitis B viral genomes. ${ }^{95}$

\section{Cancer Vaccine}

Cancer vaccines, either in the form of peptide, dendritic cellpulsed with synthetic peptide, or RNA vectors, have been used in therapeutic setting based on the principle that immune system could recognize these cancer antigens as foreign. Cancer vaccine development in HCC has been challenging and met with limited success. ${ }^{96}$ This is perhaps mostly due to the highly immunosuppressive microenvironment and highly heterogeneous HCC hampering the possibility of a single targeted cancer antigen. Several target cancer antigens for HCC include tumor-associated antigens such as NY-ESO1, glypican-3, and AFP. For instance, first HCC vaccine clinical trial against AFP showed limited clinical benefits despite detectable T-cell response. ${ }^{97,98}$ Phase I cancer vaccine studies targeting GPC-3 have proven safe and achieved posttherapeutic immunogenicity; however, clinical endpoint of relapse prevention after curative treatments (surgery or radiofrequency ablation) was not achieved in phase II studies with GPC-3. ${ }^{99,100}$

The failure of single targeted cancer vaccine could be due to the heterogeneous nature of HCC. In fact, cancer vaccines based on personalized neoantigens have demonstrated promising outcome in Melanoma patients. ${ }^{101,102}$ Currently, there is one ongoing therapeutic cancer vaccine single-arm clinical trial using therapeutic cancer vaccine IMA970A, a multipeptide-based HCC vaccine composed of 16 newly discovered and overexpressed tumor-associated peptides (TUMAPs) directly identified from resected HCC tissues (clinical trial: NCT03203005). It remains to be determined if such multipeptide cancer vaccines in HCC will be a success. However, given the immunosuppressive microenvironment of HCC, it is likely that combination with other immunotherapy will be necessary to achieve superior outcome.

Cancer vaccine based on viral antigen was designed for cancer prevention rather than therapeutic. Previous attempt to treat chronic $\mathrm{HBV}$ infection using $\mathrm{HBV}$ vaccine has failed ${ }^{78}$ and the benefit of HCV eradication in HCC was controversial. ${ }^{20-22}$ The benefit of targeting viral antigen in viralrelated HCC is therefore uncertain. On the other hand, it was also not known if any other viral-induced mutational antigens are antigenic enough for HCC tumor killing as discussed above. Therefore, cancer vaccines specific to viral-related HCC are currently not under active development. Instead, personalized cancer vaccines based on neoantigens prediction, which was tested in melanoma, ${ }^{101,103}$ could hold promising future for the treatment of HCC.

\section{Immune Checkpoint Blockade}

Upon the FDA approval of nivolumab and pembrolizumab as second-line treatment for advanced HCC, there has been an active development of immune checkpoint blockades
(ICBs) in $\mathrm{HCC}^{44,73} \mathrm{~A}$ list of the major trials involving ICBs with viral etiologies analyses is summarized in - Table $\mathbf{1}$.

\section{Anti-PD-1 Monoclonal Antibodies}

Immune checkpoints are pathways that inhibit the immune response to maintain self-tolerance and regulate the duration and amplitude of immune responses. Immune checkpoint molecules like PD-1 or PD-L1 expression are upregulated in many cancers, exploited by tumor cells to escape immune surveillance during the cancer development. In fact, it has been previously demonstrated that higher PD-1/PD-L1 is associated with poorer progression-free and overall survival after resection of primary HCC. ${ }^{104}$ Higher PD-1 expression on the CD8+ T cells is also associated with tumor progression and poor prognosis. ${ }^{48,105}$ Furthermore, attempts of using anti-PD-1 as antiviral therapy for chronic HBV infection have been described to partially restore HBVspecific CD8+ T cell functions. ${ }^{106,107}$ Based on this rationale, blocking PD-1/PD-L1 has since become a well-received immunotherapeutic option in HCC.

Two previously completed key phase I/II clinical studies in HCC using anti-PD-1 monoclonal antibodies, nivolumab, and pembrolizumab are CheckMate040 and Keynote224, respectively. ${ }^{44,73}$ CheckMate040, a phase I/II, open-label, noncomparative, dose escalation and expansion trials using nivolumab for the treatment of HCC patients previously treated with sorafenib, yielded an objective response rate (ORR) of $20 \%$ and disease control rate of $64 \% .{ }^{44}$ Interestingly, it was reported that the ORR of HBV-positive cases was lower (14\%) compared with HCV-positive (20\%) and nonviral-related cases (21-23\%) (-Table 1). ${ }^{44}$ A subsequent Asian cohort analysis demonstrated an ORR of $15 \%$ with less difference across viral etiologies (-Table 1: HBV-related ORR: 12-13\% vs. HCV-related ORR: 14-20\% or nonviral-related ORR: $13-21 \%) .{ }^{108}$ The third arm of analysis from CheckMate 040 using nivolumab and ipilimumab combination therapy has first been announced in Asco $2019^{109}$ and the analysis according to viral etiologies will be available in the near future. On the other hand, the open label phase II Keynote224 trial that assessed the efficacy and safety of pembrolizumab in patients with advanced HCC previously treated with sorafenib had demonstrated an ORR of $17 \%$ and disease control rate of $62 \%{ }^{73}$ Analysis based on hepatitis viral-related HCC: both HBV- (21\% of the total cohort) and HCV- (25\% of the total cohort) related cases again demonstrated a lower response rate in viral-related cases (ORR of 13\%) versus the uninfected cases (ORR of $20 \%)^{73}$ (-Table 1 ). 15 or $25 \%$ of the patients experienced greater than grade 3 treatment-related adverse events such as rash, pruritus, and increase in liver enzymes such as aspartate aminotransferase increase and alanine aminotransferase (ALT) from the Keynote224 and CheckMate040 trial, respectively. ${ }^{44,73}$ The rate of adverse events was as high as $37 \%$ in nivolumab and ipilimumab combination therapy. ${ }^{109}$ It is however, not known if HCC patients with viral hepatitis may experience more adverse events.

The complete reports from two phase III trials involving nivolumab (CheckMate459, NCT02576509) and pembrolizumab (Keynote240, NCT02702401) are still pending despite announcement on the failure to meet predetermined end 
points of OS. As announced by Bristol-Myers Squibb, CheckMate459 evaluating nivolumab versus sorafenib as a first-line treatment in patients with unresectable HCC did not meet its prespecified primary endpoint of OS (hazard ratio $[\mathrm{HR}]=0.85$ [95\% CI: 0.72-1.02]; $p=0.0752$, - Table 1) despite showing a clear trend toward improved OS by nivolumab compared with sorafenib, a current standard of care. The ORR according to mRECIST was reported to be around $20 \%$ similar to the outcome from CheckMate040. Keynote240, on the other hand, is a phase III, randomized, double-blind trial evaluating pembrolizumab compared with placebo in patients with advanced HCC who were previously treated with systemic therapy. According to announcement in ASCO 2019, this trial too did not meet the predetermined primary end points of improved OS (HR: 0.78; one-sided $p=0.0238$ ) and PFS (HR: 0.78 ; one-sided $p=0.0209){ }^{110}$ The ORR was however $16.9 \%$ for pembrolizumab versus $2.2 \%$ for placebo. ${ }^{110}$ The analysis based on viral-etiologies from these two phase III trials is not known at the moment, even though one would not suspect much difference compared with the results from the earlier completed phase I/II trials.

The lack of significant difference or even the inferior response rate in HBV-related versus nonviral or HCV-related HCC may be explained by several studies of TME in HCC. Our previous study comparing the TME of HBV-related versus nonviral-related HCC showed higher expression of PD-1 in HBV-related HCC. ${ }^{69}$ As PD-1 expression on CD8+ T cells was reported to be predictive of $\mathrm{PD}-1$ blockade response in nonsmall cell lungs cancer, ${ }^{111}$ one would expect a better response rate in patients with HBV-related HCC. Indeed, tumor-specific T cells were detectable in HCC patients but exist in exhausted state which could potentially be reinvigorated with checkpoint inhibitor. ${ }^{75,112}$ However, it is also important to note that the TME of HBV-HCC is enriched with Treg, ${ }^{69}$ which was previously reported to exhibit immunosuppressive phenotypes leading to disease progression and in some cases even promote hyperprogression of cancer upon PD-1/PD-L1 blockade. ${ }^{113,114}$ It therefore remains to be confirmed if a combination therapy targeting both PD-1 and Treg ${ }^{115}$ simultaneously would enhance the clinical response in HBV-related HCC particularly (-Fig. 2). Also, given the complex TME of HCC, it would also be interesting to see the possibility of further stratification of viral-related HCC to several more distinctive TMEs or molecular subgroups for better design of future immunotherapeutic strategies.

\section{Anti-PD-L1 Monoclonal Antibodies}

There are several anti-PD-L1 monoclonal antibodies: avelumab, durvalumab, and atezolizumab currently on clinical trials in advanced HCC. One phase II study of avelumab (NCT03389126) as monotherapy and another phase I study with combination of avelumab and axitinib, a small molecule tyrosine kinase inhibitor (NCT03289533), were registered and both are pending patients' recruitment. There were more trials involving the use of durvalumab, particularly a phase I/II trial in solid tumors including 39 HCC patients demonstrated a $10 \%$ ORR with better response in HCV- related cases (25\%) compared with HBV-related $(0 \%)$ and noninfected (9.5\%) $\mathrm{HCC}^{116}$ (-Table 1). This outcome is interesting and may be consistent with previous study stating a role of PD-L1 in HCV-induced immunosuppressive $\mathrm{TME}^{57,58}$ and hence supports the use of anti-PD-L1 in HCVrelated HCC (-Fig. 2). Despite that, it was also previously demonstrated that expression level of PD-L1 shows no difference between viral etiologies: HBV, HCV versus nonviral etiology in HCC. ${ }^{117}$ Another phase I/II study using the combination of durvalumab and tremelimumab (anti-CTLA4 antibody) in patients with unresectable HCC (NCT02519348) is currently ongoing and the result based on only 40 patients showed a modest $15 \%$ ORR with none of the patients with HBV or HCV-infected HCC responded ${ }^{118}$ ( - Table 1). It is not known why durvalumab alone showed better response rate in HCV-related HCC but durvalumab and tremelimumab combination therapy shows only response in noninfected HCC patients. Furthermore, the results from the above two trials involved very small cohort of patients and hence might be too immature to conclude the final verdict. We await with anticipation of the randomized, multicenter phase III study of durvalumab and tremelimumab as first-line treatment in patients with unresectable HCC: HIMALAYA study $\left(\right.$ NCT03298451) ${ }^{119}$ (-Table 1). The result from this large scale trial with estimated enrolment of 1,310 patients will be announced in the near future. The analysis based on the etiology of HCC (HBV vs. HCV vs. others) will be performed and it will be interesting to observe if there is any interesting difference between them.

Other two large-scale anti-PD-L1 combination phase III studies in advanced HCC include the COSMIC-312 trial with atezolizumab (Anti-PD-L1) + cabozantinib (multitargeted tyrosine kinase inhibitor) and the IMbrave150 trial with atezolizumab + bevacizumab (VEGF [vascular endothelial growth factor] inhibitor; - Table 1). Cabozantinib is approved as a second-line treatment in previously treated patients with advanced HCC based on improved overall survival versus placebo in the phase III CELESTIAL trial. ${ }^{120}$ COSMIC-312 (NCT03755791) is a multicenter, randomized, open-label, controlled phase III trial combining cabozantinib with atezolizumab for patients with advanced HCC who have not yet received systemic therapy. ${ }^{121}$ IMbrave150 (NCT03434379), on the other hand, is a phase III, openlabel, multicenter, randomized study to evaluate the combination of atezolizumab and bevacizumab versus sorafenib in patients with locally advanced or metastatic and/or unresectable HCC. ${ }^{122}$ It is known that VEGF plays an antiangiogenesis role which has modulatory properties on both tumor cells and the immune microenvironment. ${ }^{123}$ For instance, VEGF plays a role in Treg recruitment to the tumor and hence would be a good strategy to inhibit the pathway to enhance the local antitumor immunity. ${ }^{124}$ The IMBRAVE trial is currently ongoing with anticipation of promising outcome. In fact, with the increasing appreciation of immune-modulatory properties of targeted therapies, future combination of immunotherapy and targeted therapy based on strong rationale and well-studied mechanism of actions might be an upcoming trend. 


\section{Anti-CTLA-4 Monoclonal Antibodies}

Anti-CTLA4 antibody (ipilimumab) was first approved by FDA in 2011 for the treatment of melanoma following the phase III trial showing significant better overall survival compared with gp100 vaccine alone. ${ }^{125}$ Following that, ipilimumab as monotherapy was compared with nivolumab monotherapy or combination therapy with nivolumab in a phase III trial in advanced melanoma patients and demonstrated superior outcome in terms of both progression-free survival and median survival in combination therapy. ${ }^{126}$ This lays a foundation for combination therapy using nivolumab plus ipilimumab in HCC. The nivolumab and ipilimumab combination therapy on 148 advanced HCC patients in the third arm of analysis from CheckMate 040 was further divided to three arms with different doses and regimens. For Arm A, 50 patients were given $1 \mathrm{mg} / \mathrm{kg}$ nivolumab and $3 \mathrm{mg} / \mathrm{kg}$ ipilimumab every 3 weeks (Q3W) for four cycles, followed by $240 \mathrm{mg}$ nivolumab every 2 weeks (Q2W); Arm B, 49 patients were given $3 \mathrm{mg} / \mathrm{kg}$ nivolumab and $1 \mathrm{mg} / \mathrm{kg}$ ipilimumab Q3W for four cycles, followed by $240 \mathrm{mg}$ nivolumab Q2W; or Arm C, 49 patients were given $3 \mathrm{mg} / \mathrm{kg}$ nivolumab Q2W and $1 \mathrm{mg} / \mathrm{kg}$ ipilimumab every 6 weeks (Q6W). The initial result showing 31 to 32\% ORR with acceptable safety profile was first announced in Asco $2019^{109}$ and the analysis based on viral etiologies to this promising combination regimen will be known in the near future.

Another anti-CTLA4 antibody, tremelimumab, was previously reported in a clinical trial assessing the safety profile and antitumor as well as antiviral activity in HCV-related HCC patients (NCT01008358) ${ }^{127}$ ( - Table 1). An ORR of $17.6 \%$ based on 17 patients was reported with enhanced tumor response as well as anti-HCV viral immunity detected. ${ }^{127}$ As mentioned above, tremelimumab was also combined with durvalumab in another two more trials (NCT02519348 and NCT03298451). ${ }^{118,119}$ Given the potential benefit in anti$\mathrm{HCV}$ viral activity, the role of anti-CTLA4 ICB in HCV-related HCC warrants future validation (-Fig. 2).

\section{Future Perspectives and Concluding Remarks}

Chronic viral hepatitis infection remains one of the most common etiologies of $\mathrm{HCC}^{128}$ which will hopefully decrease with more effective control of infection in the future. In the meantime, the incidence of nonviral-related HCC particularly that from NAFLD is on the raise. ${ }^{129,130}$ Therefore, future immunotherapeutics will have to take into considerations and require deeper understanding of TME from these cases. ${ }^{131}$ In fact, our recent study implicated the role of Tim-3 in nonviral-related HCC. ${ }^{69}$ Despite the potential role of Tim-3 in T-cell dysfunction and disease progression in $\mathrm{HCC}^{55}$ there is by far only one trial in advanced primary liver cancer using TSR-022 (Anti-TIM-3 Antibody) in combination with TSR-042 (Anti-PD-1 Antibody) (NCT03680508) and its outcome will only be revealed in the future. With the increasing use and multiple combination strategies of immunotherapies as well as enhanced understanding the mecha- nisms of actions with advancement in immunomonitoring technologies, the future of treating of HCC according to various etiologies might be a possible aim to achieve.

\section{Funding}

This work was supported by the National Medical Research Council (NMRC), Singapore (ref numbers: TCR15Jun006, CIRG16may048, CSAS16Nov006, CSASI17may003, and LCG17MAY003).

Conflicts of Interest

None.

\section{References}

1 Bray F, Ferlay J, Soerjomataram I, Siegel RL, Torre LA, Jemal A. Global cancer statistics 2018: GLOBOCAN estimates of incidence and mortality worldwide for 36 cancers in 185 countries. CA Cancer J Clin 2018;68(06):394-424

2 El-Serag HB. Epidemiology of viral hepatitis and hepatocellular carcinoma. Gastroenterology 2012;142(06):1264-1273.e1

3 Abbas Z, Abbas M, Abbas S, Shazi L. Hepatitis D and hepatocellular carcinoma. World J Hepatol 2015;7(05):777-786

4 Coulie PG, Van den Eynde BJ, van der Bruggen P, Boon T. Tumour antigens recognized by $T$ lymphocytes: at the core of cancer immunotherapy. Nat Rev Cancer 2014;14(02):135-146

5 Schinzari V, Barnaba V, Piconese S. Chronic hepatitis B virus and hepatitis $C$ virus infections and cancer: synergy between viral and host factors. Clin Microbiol Infect 2015;21(11):969-974

6 Liang TJ. Hepatitis B: the virus and disease. Hepatology 2009;49 (5, Suppl):S13-S21

7 Ringelhan M, Protzer U. Oncogenic potential of hepatitis B virus encoded proteins. Curr Opin Virol 2015;14:109-115

8 Simonetti J, Bulkow L, McMahon BJ, et al. Clearance of hepatitis B surface antigen and risk of hepatocellular carcinoma in a cohort chronically infected with hepatitis B virus. Hepatology 2010;51 (05):1531-1537

9 Livingston SE, Simonetti JP, Bulkow LR, et al. Clearance of hepatitis $B$ antigen in patients with chronic hepatitis $B$ and genotypes A, B, C, D, and F. Gastroenterology 2007;133(05): 1452-1457

10 Chiu YT, Wong JK, Choi SW, et al. Novel pre-mRNA splicing of intronically integrated HBV generates oncogenic chimera in hepatocellular carcinoma. J Hepatol 2016;64(06):1256-1264

11 Furuta M, Tanaka H, Shiraishi Y, et al. Characterization of HBV integration patterns and timing in liver cancer and HBV-infected livers. Oncotarget 2018;9(38):25075-25088

12 Qasim W, Brunetto M, Gehring AJ, et al. Immunotherapy of HCC metastases with autologous $\mathrm{T}$ cell receptor redirected $\mathrm{T}$ cells, targeting HBsAg in a liver transplant patient. J Hepatol 2015;62 (02):486-491

13 Tan AT, Yang N, Lee Krishnamoorthy T, et al. Use of expression profiles of HBV-DNA integrated into genomes of hepatocellular carcinoma cells to select T cells for immunotherapy. Gastroenterology 2019;156(06):1862-1876.e9

14 Li HC, Lo SY. Hepatitis C virus: virology, diagnosis and treatment. World J Hepatol 2015;7(10):1377-1389

15 Stoll-Keller F, Barth H, Fafi-Kremer S, Zeisel MB, Baumert TF. Development of hepatitis $C$ virus vaccines: challenges and progress. Expert Rev Vaccines 2009;8(03):333-345

16 Friedman RM, Contente S. Treatment of hepatitis C infections with interferon: a historical perspective. Hepat Res Treat 2010; 2010:323926

17 Majumdar A, Kitson MT, Roberts SK. Systematic review: current concepts and challenges for the direct-acting antiviral era in hepatitis C cirrhosis. Aliment Pharmacol Ther 2016;43(12):1276-1292 
18 Chung RT, Baumert TF. Curing chronic hepatitis C-the arc of a medical triumph. N Engl J Med 2014;370(17):1576-1578

19 Cabibbo G, Celsa C, Cammà C, Craxì A. Should we cure hepatitis C virus in patients with hepatocellular carcinoma while treating cancer? Liver Int 2018;38(12):2108-2116

20 Reig M, Mariño Z, Perelló C, et al. Unexpected high rate of early tumor recurrence in patients with HCV-related HCC undergoing interferon-free therapy. J Hepatol 2016;65(04):719-726

21 Conti F, Buonfiglioli F, Scuteri A, et al. Early occurrence and recurrence of hepatocellular carcinoma in HCV-related cirrhosis treated with direct-acting antivirals. J Hepatol 2016;65(04): 727-733

22 Kozbial K, Moser S, Schwarzer R, et al. Unexpected high incidence of hepatocellular carcinoma in cirrhotic patients with sustained virologic response following interferon-free direct-acting antiviral treatment. J Hepatol 2016;65(04):856-858

23 Maan R, Feld JJ. Risk for hepatocellular carcinoma after hepatitis $C$ virus antiviral therapy with direct-acting antivirals: case closed? Gastroenterology 2017;153(04):890-892

24 Sung WK, Zheng H, Li S, et al. Genome-wide survey of recurrent HBV integration in hepatocellular carcinoma. Nat Genet 2012;44 (07):765-769

25 Cancer Genome Atlas Research Network. Electronic address: wheeler@bcm.edu; Cancer Genome Atlas Research Network. Comprehensive and integrative genomic characterization of hepatocellular carcinoma. Cell 2017;169:1327-1341

26 Hoshida Y, Villanueva A, Sangiovanni A, et al. Prognostic gene expression signature for patients with hepatitis C-related earlystage cirrhosis. Gastroenterology 2013;144(05):1024-1030

27 Abu Dayyeh BK, Yang M, Fuchs BC, et al; HALT-C Trial Group. A functional polymorphism in the epidermal growth factor gene is associated with risk for hepatocellular carcinoma. Gastroenterology 2011;141(01):141-149

28 Wang Y, Kato N, Hoshida Y, et al. Interleukin-1beta gene polymorphisms associated with hepatocellular carcinoma in hepatitis C virus infection. Hepatology 2003;37(01):65-71

29 Zong L, Peng H, Sun C, et al. Breakdown of adaptive immunotolerance induces hepatocellular carcinoma in HBsAg-tg mice. Nat Commun 2019;10(01):221

30 Rehermann B. Pathogenesis of chronic viral hepatitis: differential roles of T cells and NK cells. Nat Med 2013;19(07):859-868

31 Shimizu Y. T cell immunopathogenesis and immunotherapeutic strategies for chronic hepatitis B virus infection. World J Gastroenterol 2012;18(20):2443-2451

32 Schuch A, Hoh A, Thimme R. The role of natural killer cells and CD8(+) T cells in hepatitis B virus infection. Front Immunol 2014;5:258

$33 \mathrm{Hu}$ L, Zhai X, Liu J, et al. Genetic variants in human leukocyte antigen/DP-DQ influence both hepatitis B virus clearance and hepatocellular carcinoma development. Hepatology 2012;55 (05):1426-1431

34 Tong HV, Toan NL, Song LH, Bock CT, Kremsner PG, Velavan TP. Hepatitis B virus-induced hepatocellular carcinoma: functional roles of MICA variants. J Viral Hepat 2013;20(10):687-698

35 Gu X, Qi P, Zhou F, et al. +49G > A polymorphism in the cytotoxic Tlymphocyte antigen-4 gene increases susceptibility to hepatitis Brelated hepatocellular carcinoma in a male Chinese population. Hum Immunol 2010;71(01):83-87

36 Tang S, Yuan Y, He Y, et al. Genetic polymorphism of interleukin6 influences susceptibility to HBV-related hepatocellular carcinoma in a male Chinese Han population. Hum Immunol 2014;75 (04):297-301

37 Hoshida Y, Fuchs BC, Bardeesy N, Baumert TF, Chung RT. Pathogenesis and prevention of hepatitis $C$ virus-induced hepatocellular carcinoma. J Hepatol 2014;61(1, Suppl):S79-S90

38 Kumar V, Kato N, Urabe Y, et al. Genome-wide association study identifies a susceptibility locus for HCV-induced hepatocellular carcinoma. Nat Genet 2011;43(05):455-458
39 Bauer S, Groh V, Wu J, et al. Activation of NK cells and T cells by NKG2D, a receptor for stress-inducible MICA. Science 1999;285 (5428):727-729

40 Yarchoan M, Xing D, Luan L, et al. Characterization of the immune microenvironment in hepatocellular carcinoma. Clin Cancer Res 2017;23(23):7333-7339

41 Goodman AM, Kato S, Bazhenova L, et al. Tumor mutational burden as an independent predictor of response to immunotherapy in diverse cancers. Mol Cancer Ther 2017;16(11): 2598-2608

42 Yarchoan M, Hopkins A, Jaffee EM. Tumor mutational burden and response rate to PD-1 inhibition. N Engl J Med 2017;377(25): $2500-2501$

43 Ahn SM, Jang SJ, Shim JH, et al. Genomic portrait of resectable hepatocellular carcinomas: implications of RB1 and FGF19 aberrations for patient stratification. Hepatology 2014;60(06): 1972-1982

44 El-Khoueiry AB, Sangro B, Yau T, et al. Nivolumab in patients with advanced hepatocellular carcinoma (CheckMate 040): an openlabel, non-comparative, phase $1 / 2$ dose escalation and expansion trial. Lancet 2017;389(10088):2492-2502

45 Goossens N, Sun X, Hoshida Y. Molecular classification of hepatocellular carcinoma: potential therapeutic implications. Hepat Oncol 2015;2(04):371-379

46 Zhai W, Lim TK, Zhang T, et al. The spatial organization of intratumour heterogeneity and evolutionary trajectories of metastases in hepatocellular carcinoma. Nat Commun 2017;8:4565

47 Chew V, Chen J, Lee D, et al. Chemokine-driven lymphocyte infiltration: an early intratumoural event determining longterm survival in resectable hepatocellular carcinoma. Gut 2012;61(03):427-438

48 Chew V, Lai L, Pan L, et al. Delineation of an immunosuppressive gradient in hepatocellular carcinoma using high-dimensional proteomic and transcriptomic analyses. Proc Natl Acad Sci U S A 2017;114(29):E5900-E5909

49 Nishida N, Kudo M. Immunological microenvironment of hepatocellular carcinoma and its clinical implication. Oncology 2017; 92(Suppl 1):40-49

50 Yang P, Markowitz GJ, Wang XF. The hepatitis B virus-associated tumor microenvironment in hepatocellular carcinoma. Natl Sci Rev 2014;1(03):396-412

$51 \mathrm{Fu}$ J, Xu D, Liu Z, et al. Increased regulatory T cells correlate with CD8 T-cell impairment and poor survival in hepatocellular carcinoma patients. Gastroenterology 2007;132(07):2328-2339

52 Stoop JN, van der Molen RG, Baan CC, et al. Regulatory T cells contribute to the impaired immune response in patients with chronic hepatitis B virus infection. Hepatology 2005;41(04): 771-778

53 Yang P, Li QJ, Feng Y, et al. TGF- $\beta$-miR-34a-CCL22 signalinginduced Treg cell recruitment promotes venous metastases of HBV-positive hepatocellular carcinoma. Cancer Cell 2012;22 (03):291-303

54 Ye B, Liu X, Li X, Kong H, Tian L, Chen Y. T-cell exhaustion in chronic hepatitis $B$ infection: current knowledge and clinical significance. Cell Death Dis 2015;6:e1694

$55 \mathrm{Li} \mathrm{H}, \mathrm{Wu} \mathrm{K}$, Tao K, et al. Tim-3/galectin-9 signaling pathway mediates T-cell dysfunction and predicts poor prognosis in patients with hepatitis B virus-associated hepatocellular carcinoma. Hepatology 2012;56(04):1342-1351

56 Li FJ, Zhang Y, Jin GX, Yao L, Wu DQ. Expression of LAG-3 is coincident with the impaired effector function of HBV-specific CD8(+) T cell in HCC patients. Immunol Lett 2013;150(12):116-122

57 Nakamoto N, Cho H, Shaked A, et al. Synergistic reversal of intrahepatic HCV-specific CD8 $\mathrm{T}$ cell exhaustion by combined PD-1/CTLA-4 blockade. PLoS Pathog 2009;5(02):e1000313

58 Nakamoto N, Kaplan DE, Coleclough J, et al. Functional restoration of HCV-specific CD8 T cells by PD- 1 blockade is defined by 
PD-1 expression and compartmentalization. Gastroenterology 2008;134(07):1927-1937, 1937.e1-1937.e2

59 Piconese S, Timperi E, Pacella I, et al. Human OX40 tunes the function of regulatory $\mathrm{T}$ cells in tumor and nontumor areas of hepatitis $\mathrm{C}$ virus-infected liver tissue. Hepatology 2014;60(05):1494-1507

60 Liu P, Chen L, Zhang H. Natural killer cells in liver disease and hepatocellular carcinoma and the NK cell-based immunotherapy. J Immunol Res 2018;2018:1206737

61 Oliviero B, Varchetta S, Paudice E, et al. Natural killer cell functional dichotomy in chronic hepatitis B and chronic hepatitis C virus infections. Gastroenterology 2009;137(03):1151-1160, 1160. e1-1160.e7

62 Sun C, Sun HY, Xiao WH, Zhang C, Tian ZG. Natural killer cell dysfunction in hepatocellular carcinoma and NK cell-based immunotherapy. Acta Pharmacol Sin 2015;36(10):1191-1199

63 Boltjes A, Movita D, Boonstra A, Woltman AM. The role of Kupffer cells in hepatitis $B$ and hepatitis $C$ virus infections. J Hepatol 2014;61(03):660-671

64 Maeda S, Kamata H, Luo JL, Leffert H, Karin M. IKKbeta couples hepatocyte death to cytokine-driven compensatory proliferation that promotes chemical hepatocarcinogenesis. Cell 2005; 121(07):977-990

65 Hagen TM, Huang S, Curnutte J, et al. Extensive oxidative DNA damage in hepatocytes of transgenic mice with chronic active hepatitis destined to develop hepatocellular carcinoma. Proc Natl Acad Sci U S A 1994;91(26):12808-12812

66 Yu LX, Ling Y, Wang HY. Role of nonresolving inflammation in hepatocellular carcinoma development and progression. NPJ Precis Oncol 2018;2(01):6

67 Yeung OW, Lo CM, Ling CC, et al. Alternatively activated (M2) macrophages promote tumour growth and invasiveness in hepatocellular carcinoma. J Hepatol 2015;62(03):607-616

68 Kuang DM, Zhao Q Peng C, et al. Activated monocytes in peritumoral stroma of hepatocellular carcinoma foster immune privilege and disease progression through PD-L1. J Exp Med 2009;206(06):1327-1337

$69 \operatorname{Lim} \mathrm{CJ}$, Lee $\mathrm{YH}$, Pan L, et al. Multidimensional analyses reveal distinct immune microenvironment in hepatitis B virus-related hepatocellular carcinoma. Gut 2019;68(05):916-927

70 Aalaei-Andabili SH, Alavian SM. Regulatory T cells are the most important determinant factor of hepatitis B infection prognosis: a systematic review and meta-analysis. Vaccine 2012;30(38): 5595-5602

71 Pallett LJ, Davies J, Colbeck EJ, et al. IL-2 high tissue-resident T cells in the human liver: sentinels for hepatotropic infection. J Exp Med 2017;214(06):1567-1580

72 Sun C, Fu B, Gao Y, et al. TGF- $\beta 1$ down-regulation of NKG2D/DAP10 and 2B4/SAP expression on human NK cells contributes to HBV persistence. PLoS Pathog 2012;8(03):e1002594

73 Zhu AX, Finn RS, Edeline J, et al; KEYNOTE-224 investigators. Pembrolizumab in patients with advanced hepatocellular carcinoma previously treated with sorafenib (KEYNOTE-224): a nonrandomised, open-label phase 2 trial. Lancet Oncol 2018;19(07): 940-952

74 Hoseini SS, Cheung NV. Immunotherapy of hepatocellular carcinoma using chimeric antigen receptors and bispecific antibodies. Cancer Lett 2017;399:44-52

75 Gehring AJ, Ho ZZ, Tan AT, et al. Profile of tumor antigen-specific CD8 $\mathrm{T}$ cells in patients with hepatitis B virus-related hepatocellular carcinoma. Gastroenterology 2009;137(02):682-690

76 Fu S, Li N, Zhou PC, Huang Y, Zhou RR, Fan XG. Detection of HBV DNA and antigens in HBsAg-positive patients with primary hepatocellular carcinoma. Clin Res Hepatol Gastroenterol 2017;41(04):415-423

77 Wang Y, Wu MC, Sham JS, et al. Different expression of hepatitis B surface antigen between hepatocellular carcinoma and its surrounding liver tissue, studied using a tissue microarray. J Pathol 2002;197(05):610-616
78 Vandepapelière P, Lau GK, Leroux-Roels G, et al; Therapeutic HBV Vaccine Group of Investigators. Therapeutic vaccination of chronic hepatitis B patients with virus suppression by antiviral therapy: a randomized, controlled study of co-administration of HBsAg/ASO2 candidate vaccine and lamivudine. Vaccine 2007;25 (51):8585-8597

79 Sautto GA, Wisskirchen K, Clementi N, et al. Chimeric antigen receptor (CAR)-engineered $\mathrm{T}$ cells redirected against hepatitis $\mathrm{C}$ virus (HCV) E2 glycoprotein. Gut 2016;65(03):512-523

80 Takayama T, Sekine T, Makuuchi M, et al. Adoptive immunotherapy to lower postsurgical recurrence rates of hepatocellular carcinoma: a randomised trial. Lancet 2000;356(9232):802-807

$81 \mathrm{Xu} \mathrm{L}$, Wang J, Kim Y, et al. A randomized controlled trial on patients with or without adjuvant autologous cytokine-induced killer cells after curative resection for hepatocellular carcinoma. Oncolmmunology 2015;5(03):e1083671

82 Lee JH, Lee JH, Lim YS, et al. Adjuvant immunotherapy with autologous cytokine-induced killer cells for hepatocellular carcinoma. Gastroenterology 2015;148(07):1383-91.e6

83 Chew V, Tow C, Huang C, et al. Toll-like receptor 3 expressing tumor parenchyma and infiltrating natural killer cells in hepatocellular carcinoma patients. J Natl Cancer Inst 2012;104(23): 1796-1807

84 Ohira M, Nishida S, Tryphonopoulos P, et al. Clinical-scale isolation of interleukin-2-stimulated liver natural killer cells for treatment of liver transplantation with hepatocellular carcinoma. Cell Transplant 2012;21(07):1397-1406

85 Daher M, Rezvani K. Next generation natural killer cells for cancer immunotherapy: the promise of genetic engineering. Curr Opin Immunol 2018;51:146-153

86 Horst AK, Neumann K, Diehl L, Tiegs G. Modulation of liver tolerance by conventional and nonconventional antigen-presenting cells and regulatory immune cells. Cell Mol Immunol 2016;13(03):277-292

87 Thomson AW, Knolle PA. Antigen-presenting cell function in the tolerogenic liver environment. Nat Rev Immunol 2010;10(11): 753-766

88 Knolle PA, Thimme R. Hepatic immune regulation and its involvement in viral hepatitis infection. Gastroenterology 2014;146(05): 1193-1207

89 Howells A, Marelli G, Lemoine NR, Wang Y. Oncolytic virusesinteraction of virus and tumor cells in the battle to eliminate cancer. Front Oncol 2017;7:195

90 Jhawar SR, Thandoni A, Bommareddy PK, et al. Oncolytic virusesnatural and genetically engineered cancer immunotherapies. Front Oncol 2017;7:202

91 Yoo SY, Badrinath N, Woo HY, Heo J. Oncolytic virus-based immunotherapies for hepatocellular carcinoma. Mediators Inflamm 2017;2017:5198798

92 Park BH, Hwang T, Liu TC, et al. Use of a targeted oncolytic poxvirus, JX-594, in patients with refractory primary or metastatic liver cancer: a phase I trial. Lancet Oncol 2008;9(06):533-542

93 Heo J, Reid T, Ruo L, et al. Randomized dose-finding clinical trial of oncolytic immunotherapeutic vaccinia JX-594 in liver cancer. Nat Med 2013;19(03):329-336

94 LaRocca CJ, Warner SG. Oncolytic viruses and checkpoint inhibitors: combination therapy in clinical trials. Clin Transl Med 2018;7(01):35

95 Liu TC, Hwang T, Park BH, Bell J, Kirn DH. The targeted oncolytic poxvirus JX-594 demonstrates antitumoral, antivascular, and anti-HBV activities in patients with hepatocellular carcinoma. Mol Ther 2008;16(09):1637-1642

96 Buonaguro L, Petrizzo A, Tagliamonte M, Tornesello ML, Buonaguro FM. Challenges in cancer vaccine development for hepatocellular carcinoma. J Hepatol 2013;59(04):897-903

97 Butterfield LH, Ribas A, Dissette VB, et al. A phase I/II trial testing immunization of hepatocellular carcinoma patients with dendritic cells pulsed with four alpha-fetoprotein peptides. Clin Cancer Res 2006;12(09):2817-2825 
98 Wang X, Wang Q. Alpha-fetoprotein and hepatocellular carcinoma immunity. Can J Gastroenterol Hepatol 2018;2018:9049252

99 Tsuchiya N, Yoshikawa T, Fujinami N, et al. Immunological efficacy of glypican-3 peptide vaccine in patients with advanced hepatocellular carcinoma. OncoImmunology 2017;6(10):e1346764

100 Sawada Y, Yoshikawa T, Ofuji K, et al. Phase II study of the GPC3derived peptide vaccine as an adjuvant therapy for hepatocellular carcinoma patients. OncoImmunology 2016;5(05):e1129483

101 Ott PA, Hu Z, Keskin DB, et al. An immunogenic personal neoantigen vaccine for patients with melanoma. Nature 2017;547 (7662):217-221

102 Sahin U, Derhovanessian E, Miller M, et al. Personalized RNA mutanome vaccines mobilize poly-specific therapeutic immunity against cancer. Nature 2017;547(7662):222-226

103 Carreno BM, Magrini V, Becker-Hapak M, et al. Cancer immunotherapy. A dendritic cell vaccine increases the breadth and diversity of melanoma neoantigen-specific $\mathrm{T}$ cells. Science 2015;348(6236):803-808

104 Shi F, Shi M, Zeng Z, et al. PD-1 and PD-L1 upregulation promotes $\mathrm{CD} 8(+) \mathrm{T}$-cell apoptosis and postoperative recurrence in hepatocellular carcinoma patients. Int J Cancer 2011;128(04):887-896

$105 \mathrm{Kim} \mathrm{HD}$, Song GW, Park S, et al. Association between expression level of PD1 by tumor-infiltrating $\mathrm{CD} 8^{+} \mathrm{T}$ cells and features of hepatocellular carcinoma. Gastroenterology 2018;155(06):1936-1950.e17

106 Boni C, Fisicaro P, Valdatta C, et al. Characterization of hepatitis B virus (HBV)-specific T-cell dysfunction in chronic HBV infection. J Virol 2007;81(08):4215-4225

107 Fisicaro P, Valdatta C, Massari M, et al. Antiviral intrahepatic Tcell responses can be restored by blocking programmed death-1 pathway in chronic hepatitis B. Gastroenterology 2010;138(02): 682-693, 693.e1-693.e4

108 Yau T, Hsu C, Kim TY, et al. Nivolumab in advanced hepatocellular carcinoma: Sorafenib-experienced Asian cohort analysis. J Hepatol 2019;71(03):543-552

109 Yau T, Kang Y-K, Kim T-Y, et al. Nivolumab (NIVO) + ipilimumab (IPI) combination therapy in patients (pts) with advanced hepatocellular carcinoma (aHCC): results from CheckMate 040. J Clin Oncol 2019;37:4012-4012

110 Finn RS, Ryoo B-Y, Merle P, et al. Results of KEYNOTE-240: phase 3 study of pembrolizumab (Pembro) vs best supportive care (BSC) for second line therapy in advanced hepatocellular carcinoma (HCC). J Clin Oncol 2019;37:4004-4004

111 Thommen DS, Koelzer VH, Herzig P, et al. A transcriptionally and functionally distinct PD $-1^{+} \mathrm{CD}^{+} \mathrm{T}$ cell pool with predictive potential in non-small-cell lung cancer treated with PD-1 blockade. Nat Med 2018;24(07):994-1004

112 Flecken T, Schmidt N, Hild S, et al. Immunodominance and functional alterations of tumor-associated antigen-specific CD8+ T-cell responses in hepatocellular carcinoma. Hepatology 2014;59(04):1415-1426

113 Franceschini D, Paroli M, Francavilla V, et al. PD-L1 negatively regulates CD4+CD25+Foxp3+ Tregs by limiting STAT-5 phosphorylation in patients chronically infected with HCV. J Clin Invest 2009;119(03):551-564

114 Kamada T, Togashi Y, Tay C, et al. PD- $1^{+}$regulatory T cells amplified by PD-1 blockade promote hyperprogression of cancer. Proc Natl Acad Sci U S A 2019;116(20):9999-10008
115 Tanaka A, Sakaguchi S. Regulatory T cells in cancer immunotherapy. Cell Res 2017;27(01):109-118

116 Wainberg ZA, Segal NH, Jaeger D, et al. Safety and clinical activity of durvalumab monotherapy in patients with hepatocellular carcinoma (HCC). J Clin Oncol 2017;35:4071-4071

117 Calderaro J, Rousseau B, Amaddeo G, et al. Programmed death ligand 1 expression in hepatocellular carcinoma: relationship with clinical and pathological features. Hepatology 2016;64(06): 2038-2046

118 Kelley RK, Abou-Alfa GK, Bendell JC, et al. Phase I/II study of durvalumab and tremelimumab in patients with unresectable hepatocellular carcinoma (HCC): phase I safety and efficacy analyses. J Clin Oncol 2017;35:4073-4073

119 Abou-Alfa GK, Chan SL, Furuse J, et al. A randomized, multicenter phase 3 study of durvalumab (D) and tremelimumab (T) as firstline treatment in patients with unresectable hepatocellular carcinoma (HCC): HIMALAYA study. J Clin Oncol 2018;36: TPS4144-TPS4144

120 Abou-Alfa GK, Meyer T, Cheng AL, et al. Cabozantinib in patients with advanced and progressing hepatocellular carcinoma. $\mathrm{N}$ Engl J Med 2018;379(01):54-63

121 Kelley RK, Cheng A-L, Braiteh FS, et al. Phase 3 (COSMIC-312) study of cabozantinib (C) in combination with atezolizumab (A) versus sorafenib $(S)$ in patients (pts) with advanced hepatocellular carcinoma (aHCC) who have not received previous systemic anticancer therapy. J Clin Oncol 2019;37:TPS4157-TPS4157

122 Finn RS, Ducreux M, Qin S, et al. IMbrave150: a randomized phase III study of $1 \mathrm{~L}$ atezolizumab plus bevacizumab vs sorafenib in locally advanced or metastatic hepatocellular carcinoma. J Clin Oncol 2018;36:TPS4141-TPS4141

$123 \mathrm{Li} \mathrm{YL}$, Zhao H, Ren XB. Relationship of VEGF/VEGFR with immune and cancer cells: staggering or forward? Cancer Biol Med 2016; 13(02):206-214

124 Hansen W, Hutzler M, Abel S, et al. Neuropilin 1 deficiency on CD4+Foxp3+ regulatory $\mathrm{T}$ cells impairs mouse melanoma growth. J Exp Med 2012;209(11):2001-2016

125 Hodi FS, O'Day SJ, McDermott DF, et al. Improved survival with ipilimumab in patients with metastatic melanoma. N Engl J Med 2010;363(08):711-723

126 Wolchok JD, Chiarion-Sileni V, Gonzalez R, et al. Overall survival with combined nivolumab and ipilimumab in advanced melanoma. N Engl J Med 2017;377(14):1345-1356

127 Sangro B, Gomez-Martin C, de la Mata M, et al. A clinical trial of CTLA-4 blockade with tremelimumab in patients with hepatocellular carcinoma and chronic hepatitis C. J Hepatol 2013;59 (01):81-88

128 Stanaway JD, Flaxman AD, Naghavi M, et al. The global burden of viral hepatitis from 1990 to 2013: findings from the Global Burden of Disease Study 2013. Lancet 2016;388(10049):1081-1088

129 Blonski W, Kotlyar DS, Forde KA. Non-viral causes of hepatocellular carcinoma. World J Gastroenterol 2010;16(29):3603-3615

130 Cohen JC, Horton JD, Hobbs HH. Human fatty liver disease: old questions and new insights. Science 2011;332(6037):1519-1523

131 Karin M. New insights into the pathogenesis and treatment of non-viral hepatocellular carcinoma: a balancing act between immunosuppression and immunosurveillance. Precis Clin Med $2018 ; 1(01): 21-28$ 